\title{
PERCEPCIÓN E INFINITESIMALES EN BERKELEY $(\mathrm{I})^{*}$
}

\section{INTRODUCCIÓN}

Berkeley escribe sus Philosophical Commentaries (en adelante $P C$ ) entre los años 1707-8, esperando la oportunidad de optar por una membrecía (fellowship) en el Trinity College de Dublín. En los $P C$, Berkeley comienza a dar cuerpo y forma a su tesis inmaterialista y, al mismo tiempo, elabora un ataque detallado y brillante contra las que consideraba grandes aberraciones en el cuerpo mismo de la ciencia aceptada de su tiempo. El blanco de sus ataques era la tesis de la existencia de infinitesimales, que llegó a su florecimiento pleno con la creación del cálculo infinitesimal por Leibnitz y Newton en la segunda mitad del siglo xvir.

Berkeley dedica su energía y su agudeza mentales, en gran parte de los $P C$, al ataque y burla de la noción de infinitesimal. Considera que la expresión misma es un sinsentido y muestra, con agudeza e ingenio, que filosóficamente la noción que deseaba expresarse con ese término está plagada de problemas y confusiones y que, por esta razón, el término mismo es inútil y, por tanto, inaceptable su uso. (Estas observaciones no verán la luz pública, sino hasta casi treinta años después, cuando Berkeley publica The Analyst (1734) y crea en el mundo de la matemática una conmoción que no llegará a desaparecer sino hasta el siglo xix con la introducción, por Weierstrass y otros, de la noción de límite y la eliminación de los infinitesimales de los fundamentos mismos del cálculo. Pero, ciertamente, la historia no acaba ahí; el teorema de Skolem (1934) sobre modelos no estándar de la aritmética y el desarrollo del cálculo no estándar de Robinson han introducido nuevamente al mundo del ser - aun cuando con cartas credenciales muy distintas a las que tenían en el cálculo leibnitziano- a los infinitesimales que el obispo Berkeley rechazara con tanta lucidez en su alegato, hace ya más de doscientos años.)

Dada la concepción que Berkeley tenía de la noción de infinitesimal, su opinión (aun cuando ésta permaneció, en sus puntos más virulentos, privada, figurando tan sólo en los $P C$ ) acerca de quienes eran dados a emplearla, a saber, los matemáticos, fue de burla y desprecio:

Si se acusa a las Disputas de los Escolásticos de intrincadas frívolas y confusas, debe reconocerse sin embargo que básicamente trataban temas grandes e importantes. Si admiramos el Método y la agudeza de los Mat:

* La segunda y última parte de este trabajo (capítulo del libro en preparación Percepción, aritmética y geometria en George Berkeley) aparecerá en Diánoia 1981. 
la longitud, la sutileza, la exactitud de sus Demostraciones, nos vemos sin embargo forzados a conceder que en su mayor parte son acerca de temas frívolos y quizás acerca de nada en absoluto. ( $P C 449$ )

Hemos aprendido de Mr. Locke. que hay muchos Discursos verbosos, coherentes, metódicos que sin embargo no valen nada. él insinúa esto con respecto a los Escolásticos. Nosotros podemos aplicarlo a los Matemáticos. ( $P C$ 492)

Únicamente Newton se salva de esta opinión:

No veo ingenio en ninguno de ellos sino en Newton, El resto son meramente charlatanes, puramente Nihilistas. ( $P C$ 372)

Algunos Matemáticos tienen buenas dotes, la mayoría es lamentable. De no haber sido Matemáticos habrían sido buenos para nada. fueron tales tontos que no supieron cómo emplear sus dotes. (PC 375) ${ }^{1}$

1 En 1726, Jonathan Swift, quien fuera compatriota, gran amigo de Berkeley y que, además, comparte las opiniones de éste, según veremos en un momento, en el "Viaje [de Gulliver] a Laputa", habla de los matemáticos y músicos, habitantes de esta curiosa isla, de la siguiente manera:

...although they are dextrous enough upon a piece of paper in the management of the rule, the pencil and the divider, yet in the common actions and behaviour of life, I have not seen a more clumsy, awkward, and unhandy people nor so slow and perplexed in their conceptions upon all other subjects, except those of mathematicks and musick ... Imagination, fancy, and invention, they are wholly strangers to, nor have any words in their language by which those ideas can be expressed... ([18] p. 197.)

$\mathrm{Y}$, para concluir la sátira, Swift añade aún:

The women of the island have abundance of vivacity; they contemn their husbands, and are exceedingly fond of strangers, whereof there is always a considerable number... Among these the ladies chuse their gallants: but the vexation is, that they act with too much ease and security; for the husband is so wrapped in speculation, that the mistress and lover may proceed to the greatest familiarities before his face, if he be but provided with paper and implements, and without his flapper at his side. (Ibid., p. 199.)

Aun cuando Berkeley nunca llegó a expresarse de esta manera, tanto él como su amigo Swift llegaron, sin embargo, a la misma conclusión: es preferible dedicarse a cosas más nobles que la matemática (aun cuando Swift podría haber añadido, quizá con maliciosa sonrisa: o bien, en caso contrario, siempre es prudente hacer voto de castidad). -Moulines fue quien me puso en camino de encontrar estas malévolas observaciones de Swift, que añaden un poco de pimienta al texto.

Ya que aquí hemos mencionado a Swift, quizá sería interesante, para quienes son dados a este tipo de consideraciones, saber que su cerebro está catalogado como uno de los más masivos de los que se tiene noticia (se considera que su volumen rebasaba los $2000 \mathrm{cc}$ ), junto con los de Cromwell, Turguéniev y Lord Byron; sin embargo, el volumen del cerebro no puede considerarse un dato fundamental en lo que toca a las facultades mentales de su poseedor, ya que el cerebro de Anatole France apenas si excedía los $1000 \mathrm{cc}$. 
Ahora bien, en sus escritos publicados, Berkeley observó el precepto que se impuso en los mismos $P C$ :

Mem: en todas las ocasiones usar la máxima modestia. Refutar a los matemáticos con extrema cortesía y respeto. No llamarlos nihilistas etc: (PC 633)

$y$, de inmediato, añade:

N.B. refrenarte en tu naturaleza Satírica (PC 634)

Una de las consecuencias de la doctrina de los infinitesimales que más ofendía a Berkeley era que, conforme a la misma, cualquier extensión, por grande o pequeña que fuera, podía dividirse infinitamente para obtener, de ella, partes infinitesimae, y no sólo eso sino que, además, podian hacerse, conforme a tal doctrina, divisiones infinitamente pequeñas de las entidades ya infinitamente pequeñas a las cuales se había llegado con la primera división, obteniéndose de esta manera los infinitesima infinitesimarum, y así sucesivamente.

- Berkeley encuentra incomprensible la doctrina. El mayor problema que la misma le presenta en el campo de la percepción es que, conforme a ella, cualquier objeto perceptual extenso estaría compuesto de un infinito número de partes, imperceptibles todas y, así, ajenas por completo a la posibilidad de ser conocidas o de tener existencia (conforme a su principio esse est percipi).

En las páginas que siguen, consideraré los argumentos de Berkeley en contra de la doctrina de los infinitesimales sólo en lo que concierne a la tesis, que de la misma se sigue, de la divisibilidad infinita de los objetos de la percepción. Este punto, en general, no lo he visto tratado en la literatura berkeleyana y considero que nos permite conocer otro aspecto de los sutiles y, a menudo, brillantes argumentos del que fuera obispo de Cloyne.

Por otro lado, la mayoría de las tesis que aquí discutiré están formuladas en los Philosophical Commentaries y muchas de ellas no vieron la luz pública, cuando menos elaboradas en detalle (me refiero concretamente a la tesis de la constitución de los objetos de la vista y del tacto en términos de minima sensibilia), aun cuando ciertamente estaban vivas en la mente de Berkeley cuando éste redactó su Teoria de la visión (TV; en esta obra los minima visibilia se discuten en las secciones 54, 79-83) y sus Principios (PCH; en esta obra, los minima sensibilia figuran en la sección 132) y esperaban, posiblemente, recibir una elaboración mayor en un libro que fuera continuación de la Parte I (única publicada) de los Principios; en efecto, en PC 583 y 853 Berkeley menciona tres libros en proyecto, aun cuando posteriormente, en su correspondencia, señala que perdió el manuscrito de la continuación de los Principios. 
Considero que el que las tesis de Berkeley referidas a estos puntos hayan quedado inéditas no les quita importancia pues, tal como aparecen las anotaciones en los $P C$, parecen formar una parte central de su filosofía; y lograr que se discutan nos permitirá tener una idea más cabal y plena de uno de los filósofos más estimulantes y merecedores de atención.

\section{PERCEPGION E INFINITESIMALES}

La teoría general que Berkeley defiende acerca de los objetos extensos de percepción es que éstos no son infinitamente divisibles, pese a la creencia común entre los matemáticos de su época. La posición que Berkeley ataca podría formularse en los siguientes términos:

Cualquier objeto extenso de percepción, por grande o pequeño que sea, puede descomponerse en (o está compuesto de) una(s) serie(s) infinita(s) de puntos.

Berkeley encuentra que esta suposición, por una parte, es paradojica (ya que implica las llamadas "paradojas del infinito") y así nos dice:

Mem. probar en contra de Keil que la divisibilidad infinita de la materia hace que la mitad tenga igual número de partes iguales que el todo. (PC 322)

y, además,

Keil llena el mundo con un ácaro (mite) esto se sigue de la Divisibilidad ad infinitum de la extensión. (PC 364) ${ }^{1}$

2 Luce, en [3], pp. 379-380, nos dice lo siguiente acerca de Keill:

John Keill F.R.S. (1671-1721) enseñó física y geometría en Oxford y fue nombrado posteriormente profesor de astronomia; fue "el primero que enseñó filosoffa natural mediante experimentos a la manera matemática" (cita de Dict. Nat. Biogr.). Escribió An Examination of Dr. Burnet's Theory of the Earth (1698), Introductio ad Veram Physicam (1702) y diversos articulos sobre fluxiones en las Philosophical Transactions. Redactó, para la Royal Society, el Commercium Epistolicum en el que se le daba prioridad a Newton sobre Leibniz en el descubrimiento del cálculo. Berkeley tenía en mente las Lectiones IV, V, de la Introductio.

No fue sino hasta el siglo xIx, con Cantor, cuando se aclaró la noción del infinito y se eliminaron las supuestas paradojas que la misma parecia encerrar; pero esto sucede siempre y cuando aceptemos ciertos supuestos que Berkeley, junto con los matemáticos intuicionistas contemporáneos, no estaria dispuesto a aceptar. El mayor de todos ellos es suponer que hay, al menos, una totalidad infinita, un conjunto infinito de puntos. Una vez aceptado esto se puede, utilizando las técnicas que Cantor descubrió y desarrolló, generar una jerarquía de 
Por otra parte, la tesis de la divisibilidad infinita de la materia va en contra del principio central (esse est percipi) de la filosofía de Berkeley, lo cual da a éste otro motivo - y de suma importancia, como es obvio- para oponerse a una tesis semejante. Así, en algunas de las primeras anotaciones de los $P C$, Berkeley escribió lo siguiente:

La extensión no es infinitamente divisible en un sentido. ( $P C$ 11)

La extensión una sensación, por tanto no sin [fuera de] la mente. (PC 18)

La demostración de la divisibilidad infinita de la extensión supone longitud sin ancho que es absurdo. ( $P C$ 21)

extensión invisible (PC 21a)

La divisibilidad infinita de la extensión sí supone la existencia externa de la extensión pero lo último es falso, ergo también lo primero. ( $P C 26$ )

Que exista la extensión en una cosa no pensante es una contradicción (PC 37)

o más bien en una cosa carente de percepción. Pareciendo que el pensamiento implica acción. (PC 37a)

Estos pasajes de Berkeley muestran, con bastante claridad, la razón por la cual se niega a aceptar la divisibilidad infinita del espacio; a saber, porque ésta supone que la extensión es algo que puede tener ser fuera de la mente y esto, conforme a Berkeley, es absurdo. Tal absurdo surge de la premisa (PC 18) de Berkeley: "La extensión una sensación..." y, puesto que ninguna sensación puede existir sin una mente que se haga consciente de tal sensación, Ia extensión sin la mente no es, no tiene ser. Por otra parte Berkeley, en las anotaciones que hemos citado, presenta otra razón para rechazar la divisibilidad infinita: tal divisibilidad supone que puede haber una cosa tal como extensión sin ancho o, en otras palabras, una pura extensión generada por un punto matemático que no tiene dimensión alguna. Estas dos nociones, las de la línea y el punto matemáticos, Berkeley las rechazará por dos razones:

a) la señalada en los pasajes citados, esto es, porque ninguna de ellas se refiere a algo que pueda ser el objeto de una sensación; posteriormente,

ordenes de infinitud en la que hay diferentes "tamaños" de infinitos. No hay que considerar, sin embargo, la teoria de Cantor como una descripción del universo; esto trae consigo serias confusiones. Quien desee enterarse de cómo puede confundirse acerca de esto una mente lúcida, puede ver [1], pp. 75-89 y, para un comentario a este texto, [13], pp. 49-54. 
en una etapa más avanzada de su meditación sobre estos temas, Berkeley añadirá

b) que estas nociones aluden a ideas abstractas que él considera que son algo imposible, algo que implica una contradicción.

Por lo tanto, la conclusión que Berkeley alcanza, sea por la razón $a$ ) o por la razón $b$ ), es que las nociones que aquí estamos considerando no aluden a nada; las palabras que las expresan no tienen contenido alguno, por lo que nos engañamos pensando que algo nos comunican. Ciertamente, Berkeley llegó a expresar:

Axioma. No usar ninguna palabra sin una idea. ( $P C$ 356)

y aun cuando luego modificó esta afirmación - pues llegó a convencerse de que no teníamos idea de cierto tipo de entidades que, sin embargo, debíamos aceptar en nuestra ontologia-, la idea que en ella se expresa es una que tuvo presente durante la redacción de la primera parte de sus $P C$. La misma idea, enfocada a un blanco especifico, la habia expresado, unas cuantas líneas atrás, de la siguiente manera:

Axioma. Ningún razonamiento acerca de cosas de las que no tenemos idea alguna. Por tanto, ningún razonamiento acerca de infinitesimales. $(P C$ 354)

Teniendo en cuenta lo que hasta ahora he señalado, en lo que sigue presentaré y desarrollaré los siguientes temas:

1. Tesis con las que se pretende fundar la divisibilidad infinita. Bajo este rubro consideraré los siguientes tres apartados:

(1) Existencia de series infinitas de puntos en la composición de cualquièr objeto extenso.

(2) Argumento del microscopio.

(3) Argumento de la atención.

II. Réplica de Berkeley a las tesis anteriores. Consideraré lo siguiente:

(1) Composición finita, discreta (discontinua) de los objetos (de percepción) extensos.

(i) Rechazo de la argumentación matemática como aplicable a los objetos (de percepción) extensos. 
(ii) Naturaleza y composición del objeto (de percepción) extenso: minima sensibilia (m.s.; esta expresión comprende los minima visibilia — m.v._ así como los minima tangibilia - m.t.). Dentro de este apartado considero las tesis de Locke acerca de la constitución del objeto material, pues así podrá verse con mayor claridad la reacción de Berkeley contra estas tesis y considero que se obtiene una mejor visión de su propia posición positiva.

(2) Heterogeneidad de los objetos de percepción percibidos en tiempos o por sentidos diferentes.

\section{Divisibilidad INFInIta}

(1) Series infinitas (SI)

La tesis bajo este rubro en contra de la cual argumentará Berkeley, él mismo la formula en los siguientes términos:

(A) Si cada partícula de materia es extensa debe ser infinitamente extensa. o tener una serie infinita de extensión. ( $P C$ 67)

y la misma, más matizada, la presenta en los Principios de la siguiente manera:

...debe señalarse que actualmente, cuando menos los filósofos más distinguidos y connotados, conceden universalmente la divisibilidad infinita de la materia, y la demuestran más allá de toda duda conforme a los principios aceptados. Por tanto, se sigue que hay un número infinito de partes en cada partícula de materia, que no son percibidas por los sentidos. $(P C H$, sec. 47$)$

Una vez señalado lo anterior, paso a formular un principio general adecuado, a mi entender, para fundar la que he denominado proposición $(A)$ de Berkeley:

$\left(A_{1}\right)$ Todo lo extenso debe estar compuesto de partes extensas ya que, de otra manera, lo no extenso formaría la extensión, lo cual es contradictorio. $^{2}$

2 En Euclides, Elementos, x, escol. i (cf. [6], pp. 215-6), encontramos ya una proposición en el sentido en el que apunta mi $\left(A_{1}\right)$ :

...en resumen, la magnitud, en la división, participa del principio de lo infinito 
(A) se deriva con bastante facilidad de $\left(A_{1}\right)$ ya que:

1. Hay partículas extensas de materia (premisa perceptual).

2. Cada partícula extensa de materia debe estar formada de partes extensas (por $\left.\left(A_{1}\right)\right)$;

pero entonces,

3. Cada una de estas partes extensas estará formada... (nuevamente por $\left.\left(A_{1}\right)\right)$.

y así sucesivamente, ad infinitum.

Intuitivamente, el principio $\left(A_{1}\right)$ parece claro y plausible. ¿Cómo sería posible que de lo no extenso pudiera brotar la extensión? No pensemos, por el momento, en términos de puntos y líneas matemáticos que corresponden, justamente, al mundo de la abstracción matemática, acerca del cual no diremos demasiado en lo que sigue; pensemos en el mundo de puntos y líneas físicos. ${ }^{3}$

De tales líneas y puntos físicos podemos, sin esforzarnos demasiado, aceptar que si tienen extensión, por pequeña que ésta sea, será posible, en principio, bisectarlos y obtener, así, p. ej., dos mitades del punto dado, las que, a su vez, deben ser capaces de recibir una nueva bisección. Una vez que aceptamos esto, sin embargo, nos hemos embarcado en un proceso sin fin de bisecciones, mismo en el que Zenón embarcó a Aquiles y donde éste sigue aún intentando alcanzar a la huidiza tortuga.

Visto de otra manera, lo que nos señala el principio $\left(A_{1}\right)$ es que de la negación de la extensión no puede obtenerse la extensión $o$, dicho matemáticamente, que el cociente de las sucesivas divisiones a las que sujetemos a la unidad de materia dada nunca puede ser 0 pues, siendo así, nuestro punto de

pero, en su totalidad, del principio de lo finito; en tanto que el número participa, en la división, de lo finito pero, en su totalidad, de lo infinito...

La historia de este principio es, pues, bastante larga. Para los griegos, al menos de Euclides en adelante, la geometría era una ciencia de las cantidades continuas y la aritmética lo era de las discontinuas o discretas. Berkeley rechazará la tesis continuista con respecto a la geometría e intentará ver a ésta desde una perspectiva de aritmética discreta. Cf., además, n. 8.

3 Para aclarar un tanto este pasaje puedo señalar que, si alguien argumentara que el movimiento de un punto matemático (no extenso) es el que produce la línea (longitud sin ancho), el movimiento de la línea produce el plano (extensión sin espesor) y el movimiento del plano produce los cuerpos, esto sería una petitio en contra de Berkeley, ya que él cuestiona, justamente, que un punto totalmente inextenso y, por tanto, imperceptible, pueda generar objetos perceptuales. La presentación y discusión de estos aspectos de la filosofia de Berkeley tendrá que esperar una segunda parte de este trabajo, en la cual se expondrán las tesis positivas acerca de lo que Berkeley consideraba el objeto propio de estudio de la geometría, rechazando en base a esto las que consideraba tesis abstraccionistas de los matemáticos o geómetras de su época. 
partida debería haber sido, también, 0. Esto es, no hubiésemos tenido punto de partida, lo cual contradice nuestra hipótesis.

Si nos quedamos con una formulación como la anterior acerca de la divisibilidad de la materia - y, según veremos, no tenemos por qué esforzarnos en ir más a fondo en esta cuestión, cuando menos en el contexto de la discusión presente-, nos encontraremos con que cualquier punto, por extremadamente pequeño (no infinitamente pequeño) que sea, al que lleguemos en nuestro proceso de división, puede emplearse como una unidad generadora de la extensión original si es que le añadimos un número de unidades suficientemente grande (no infinito) como para alcanzarla. Hasta este punto podemos afirmar que los trozos terriblemente pequeños de extensión que aquí consideramos, cumplen fielmente con su correspondiente principio arquimediano, esto es, siempre podremos alcanzar, con ellos, cualquier extensión dada, por grande que se la suponga, si tenemos un número suficiente (no infinito) de tales trozos terriblemente pequeños de extensión.

Aquí parece que puede formularse la siguiente objeción: una vez supuesto todo lo anterior, no se ve nada que nos impida proseguir al infinito el proceso de división, con lo cual, obtendriamos puntos tan insignificantes en extensión (puntos infinitesimales, diferentes de 0) que carecerian de la propiedad arquimediana a la que arriba se ha aludido. ¿Por qué hemos de parar en algún momento nuestro proceso de división antes de alcanzar tales puntos?

La respuesta que daré a la pregunta anterior aclarará y precisará, según espero, los límites de lo que aquí quiero discutir, evitando así falsas expectativas por parte de los lectores que hayan llegado conmigo hasta este punto. Por una parte, la objeción postula un infinito actual, esto es, un conjunto infinito de divisiones ya efectuadas sobre nuestro objeto extenso; Berkeley está dispuesto a reconocer, en un sentido que ya precisaremos, un infinito potencial y rechaza, enfáticamente, la posibilidad (lógica) misma de uno actual ${ }^{4}$

4 En una época simultánea o ligeramente posterior a la redacción de los $P C$, según nos dice Luce en [4], vol. IV, pp. 233-4, Berkeley escribió el ensayo "Of Infinites", en el que señala:

Estoy díspuesto a creer que fácilmente podría ponerse fin a estas disputas y escrúpulos, surgidos del uso que se hace, en los métodos antes mencionados, de cantidades infinitamente pequeñas, con la sola consideración de un pasaje del incomparable tratado de Mr. Locke sobre el Entendimiento humano, 1. 2, cap. 17, sec. 7, donde ese autor, manejando el tema de la infinitud con ese juicio y claridad que le son tan peculiares, tiene las siguientes palabras:

sospecho que producimos gran confusión en nuestros pensamientos cuando unimos la infinitud a cualquier supuesta idea de cantidad que se piense que la mente puede tener y de esta manera disertamos o razonamos acerca de una cantidad infinita como, por ejemplo, un espacio infinito o una infinita duración. Porque nuestra idea de infinitud, según creo, es una idea en desarrollo sin fin, pero la idea que la mente tiene de cualquier cantidad está en ese momento terminada en esa idea; añadirle la infinitud es tratar de ajustar una me- 
-no deseo entrar aquí en una discusión acerca de estos puntos, ni me parece necesaria en el contexto presente. Por otra parte, según veremos, Berkeley se niega a aceptar incluso la posibilidad de una indefinida (no digamos ya infinita) serie de partes en las cuales puedan dividirse los objetos extensos perceptibles. Para él, cualquier objeto así tiene ya un número fijo, determinado, de partes últimas y será esto, justamente, lo que producirá el conflicto entre la posición hasta aquí presentada y la suya propia. Finalmente, puedo señalar otra razón que Berkeley, según veremos, tiene muy en cuenta para rechazar una objeción como la que aquf se comenta, y es que en tal objeción, según la he formulado, se confunden los que podriamos denominar planos teórico y físico; esto es, se supone que la divisibilidad puede, físicamente, realizarse hasta obtener un número infinito de partículas, lo cual es imposible; suponer tal infinitud de partículas sólo puede hacerse en el plano teórico. ${ }^{5}$ Por lo anterior, no creo que sea preciso abundar aquí en los problemas del infinito; espero que lo dicho se aclarará una vez que veamos estas últimas observaciones desarrolladas en el contexto de la réplica de Berkeley. ${ }^{6}$

dida fija a una cantidad creciente. Por esa razón no creo que sea una sutileza sin sentido decir que debemos distinguir cuidadosamente entre la idea de la infinitud del espacio y la idea de un espacio infinito.

Ahora bien, si lo que dice Mr. Locke se aplicase, mutatis mutandis, a las cantidades infinitamente pequeñas, esto nos libraría, sin duda, de la oscuridad y confusión que de otra manera entorpecen muy grandes avances en el Análisis Moderno. ([4], vol. IV, p. 235. Véase, además, infra, n. 11.)

5 Para un comentario y una precisión acerca del alcance de la distinción a la que aquí 'aludo, véase n. 20 (en la segunda parte de este trabajo).

6 Vale la pena aclarar aquí un punto que ha causado cierta confusión en algunos de mis lectores: Berkeley considera que la tesis de la divisibilidad infinita afirma, de manera tajante, que dado un objeto extenso, $A$, es posible (si no empíricamente sí, al menos, en principio) bisectarlo y hacer lo mismo con las partes resultantes, $A_{1}$ y $A_{2}$, y repetir la operación con las nuevas partes resultantes, $A_{1_{1}}, A_{1_{2}}, A_{2_{1}}$ y $A_{2_{2}}$, etc., hasta obtener fragmentos infinitamente pequeños (¿aún extensos?) como resultado de estas continuas bisecciones. Estos fragmentos, sin embargo, habrian perdido, ya, una de las propiedades del fragmento $A$ que los originó, a saber, la de poder alcanzar una extensión (finita), por pequeña que ésta fuera, mediante la adición de un número finito, por grande que éste sea, dé tales partículas. Aquí podemos detenernos y preguntar si es posible hacer que nuestro concepto de extensión se aplique a partículas infinitesimales; ¿tenemos algún criterio de aplicación de tal concepto a niveles semejantes de pequeñez? $Y$ quizá no tengamos que descender tanto en la escala de "tamaño" (que también tiene un campo de aplicación más o menos bien delimitado) para formularnos una pregunta asi; podríamos hacerlo, incluso, acerca de las partículas que, conforme a los físicos, forman los nucleones atómicos: ¿podemos aplicar a éstas nuestros conceptos (normales) de tamaño, extensión, etc.? Pero detengamos aquí estas observaciones y volvamos con nuestro tema. La conclusión a la que llegaría quien sostuviera una tesis como la de la divisibilidad infinita antes mencionada, sería que, como el número infinito de partículas infinitesimales se obtuvo del objeto $A$, éste está formado por un número infinito de "partes", las partículas infinitesimales de las que aqui hablamos.

Berkeley rechaza que lo anterior sea siquiera posible en principio. No hay sino un número finito de partes formando, realmente, cualquier objeto. Además, si se nos dice que 
Resumiendo: la tesis que Berkeley combatirá acerca de la divisibilidad infinita de la materia, puede verse como fundada en un principio que parece, en algún sentido, bastante plausible (o que, al menos, pudo parecer plausible a los contemporáneos de Berkeley y, quizás, a él mismo en algún momento ${ }^{7}$ ) y que establece que la extensión no puede generarse de lo que no la tiene.

\section{(2) Argumento del microscopio (AM)}

Lo que con este argumento se intentará mostrar es que uno y el mismo objeto extenso está formado por innumerables partes que escapan a nuestra detección a simple vista, pero que es posible percibir apelando a la ayuda de lentes de aumento o de microscopios.

Ahora bien, si el $A M$ es un buen argumento, vendrá en ayuda de la tesis SI, de la siguiente manera: si, según sabemos, una de las tesis conocidas de Berkeley es que no hay objetos de percepción que existan sin ser percibidos, entonces el $A M$ podría mostrar que hay partes minúsculas de entidades macroscópicas: partes minúsculas que, conforme a $S I$, deben existir. De esta mane-

Berkeley niega que cualquier objeto, $A$, tenga actualmente un número infinito de partes pero aceptaría, quizás, que potencialmente las tuviera, quiero señalar que no es claro, en manera alguna, lo que esto quiere decir. Pero lo que si puede afirmarse acerca de la tesis de Berkeley es que, para él, ningún objeto actual es infinito (entendiendo por esto la posibilidad de dividirlo infinitamente) ni actual ni potencialmente. Lo que puede suceder es que el objeto $A$ represente a cualesquiera objetos de su tipo, por grandes o pequeños (finitamente grandes o finitamente pequeños) que pueden ser y es en tanto que representante (no en tanto que objeto actual $A$ ) como podríamos suponer que pudiera dividirse en más partes (pero éstas, siempre en número finito) de aquéllas en las que en realidad puede divi. dirse. Algunos de estos puntos se presentarán y discutirán más adelante en el texto principal.

7 Berkeley, en "Of Infinites" (cfr. supra n. 4), escribió lo siguiente:

A mí me es claro que no debemos usar signo alguno sin una idea que responda al mismo; y muy claro es que no tenemos idea alguna de una línea infinitamente pequeña; más aún, es evidentemente imposible que pueda haber cosa tal, pues cualquier línea, por pequeña que sea, es divisible aún en partes menores que ella misma; por tanto, no puede haber una cosa tal como una línea quavis data minor o infinitamente pequeña. (loc. cit. pp. 235-6.)

De este pasaje parece seguirse claramente que Berkeley concede que un principio como nuestro $\left(A_{1}\right)$ es verdadero y, sin embargo, se niega a concluir del mismo que hay un número infinito de partes en una línea cualquiera. Estas tesis pueden perfectamente hacerse consistentes señalando que Berkeley está aquí considerando un proceso efectivo de división, esto es, un proceso que prácticamente podamos efectuar $\mathrm{y}$, ciertamente, nunca podremos alcanzar un número infinito de puntos haciendo divisiones en un segmento de recta. Berkeley, entonces, rechaza en este pasaje la infinitud porque no es algo que podamos alcanzar en la práctica. No será sino más adelante, en una etapa más avanzada de su pensamiento, pero aún en los $P C$, cuando hará una precisión todavía mayor acerca de tales tesis: hay un momento en el que no es posible (ni fisica ni teóricamente) hacer una división más en cualquier objeto extenso, y este momento llega cuando la presentación que tenemos del objeto extenso es la de un minimum sensibile (m.s.). 
ra, el $A M$ puede considerarse un argumento que viene a dar cierto tipo de apoyo empírico a lo que $S I$ nos dice que, en principio, debe existir.

Dicho en otros términos, si Berkeley objetase que $S I$ es inadmisible porque no son perceptibles los minúsculos trozos de extensión que, conforme a SI, debe haber en cualquier objeto extenso, entonces el $A M$ pondría a la vista, por así decirlo, tales elementos minúsculos.

Según esto, entonces, una gota de sangre vista con el ojo desnudo y esta misma gota vista a través del microscopio, muestran aspectos completamente diferentes, ya que en la última distinguiremos una enorme variedad de elementos constitutivos de la gota que no podíamos percibir cuando a ésta la observábamos sin ayuda del microscopio. La conclusión a la que llegaría alguien que sostuviese el $A M$ sería, pues, que la gota de sangre está constituida por elementos imperceptibles (a simple vista) que son parte de la misma.

Una vez presentadas las consideraciones intuitivas anteriores, podemos formular el argumento del microscopio en los siguientes términos:

( $A M)$ Puesto que el microscopio nos muestra partes microscópicas, imperceptibles a simple vista, del objeto macroscópico, éste está constituido por tales partes microscópicas; esto es, está constituido por partes imperceptibles a simple vista.

Si ahora consideramos una aplicación reiterada del $A M$ y pensamos que el mismo vale para las mismas partes microscópicas —en las cuales, mediante el empleo de lentes más potentes, podrían descubrirse partes aún más pequeñas - y esta aplicación la prolongamos ad indefinitum, podemos concluir que cada objeto de percepción sucesivo está conformado por partes imperceptibles en ese nivel de observación, pero que podrán tornarse perceptibles mediante el uso de aparatos cada vez más potentes. Así, la conclusión a la que aquí se llega es que el $A M$ muestra que cualquier objeto extenso está formado por un número indefinidamente grande (al menos) de partes extensas; y si a esto agregamos el principio $\left(A_{1}\right)$ de $S I$, la conclusión de la divisibilidad infinita es inmediata.

Berkeley, comentando el argumento de la divisibilidad infinita en términos de lentes de aumento (o el $A M$ que aquí consideramos), dice:

Las líneas se cree que son Divisibles ad infinitum porque se supone que existen fuera. También porque se cree que son las mismas cuando se las ve con el ojo desnudo y cuando se las ve con lentes de aumento. (PC 236)

Resumiendo el anterior apartado, podemos decir que el $A M$ se presenta básicamente como un argumento que viene a reforzar lo que, en principio, afirma SI. De esta manera lo toma Berkeley al decirnos: 
Quienes no conocían las Lentes no tenían una excusa tan favorable para la Divisibilidad ad infinitum. (PC 237)

Además, el $A M$ puede verse como fundando una tesis de existencia de entidades no perceptibles, en tanto que el mismo se aplique reiteradamente un número indefinidamente grande de veces.

\section{(3) Argumento de la atención $(A A)$.}

El $A A$ es muy similar al $A M$ y lo que con él se pretende es, una vez más, señalar que un objeto puede estar constituido por partes que no se captan a primera vista, sino una vez que el objeto se analiza con cuidado y atención. Así, de este argumento se concluye que en uno y el mismo objeto puede haber elementos que lo constituyen y que pueden pasar desapercibidos en una primera observación.

Berkeley, en los $P C$, toma en cuenta la posibilidad de un argumento así cuando señala:

Pr. si la extensión de un plano visto directamente y de manera oblicua, examinado minuciosa y distintamente o a la vez de Bulto y confusamente, es la misma.

N.B. se supone que el plano conserva la misma distancia. (PC 283)

y, más adelante, añade:

Pr: si no hay dos clases de extensión visible, una percibida mediante una visión confusa, la otra mediante una dirección distinta sucesiva del eje óptico a cada punto. (PC 400)

En el caso que aquí he presentado, puedo concluir lo siguiente: tanıo el $A M$ como el $A A$ tienen una intención similar, a saber, cuestionar que un objeto de percepción se presente totalmente ante el perceptor. Habrá aspectos de aquél que no sean considerados por éste en una primera aproximación $(A A)$ o, incluso, que no puedan (de hecho) ser considerados por el perceptor sin apelar a algún artificio óptico: lentes de aumento o microscopios $(A M)$. Tanto en el caso del $A M$ como del $A A$, se abre la posibilidad de que la percepción de determinado objeto perceptual (extenso) quede incompleta y, en el caso del $A M$, aquélla sería incompletable. Siempre habría la posibilidad de apelar a lentes más potentes que nos descubrieran nuevos elementos en los objetos de observación. 


\section{RÉPlica DE BeRKeley}

(1) Composición finita del objeto extenso de percepción

Primeramente consideraré un argumento de Berkeley en contra de la divisibilidad infinita:

(a) Divisibilidad finita del objeto perceptual. En pocas palabras, el argumento de Berkeley consiste en señalar que los matemáticos han confundido el campo de aplicabilidad de sus argumentos y esto les ha producido la ilusión de que los objetos perceptuales extensos son infinitamente divisibles,

para luego ocuparme de los aspectos constructivos de la tesis de Berkeley:

(ß) Composición finita, discreta (discontinua) del objeto perceptual. El objeto extenso está formado por una cantidad discreta, finita, de átomos de percepción, los minima sensibilia; en este apartado trataré (a) la tesis de Locke acerca de la constitución del objeto material y (b) la réplica de Berkeley y su tesis positiva a este respecto.

( $\gamma$ ) Heterogeneidad del objeto perceptual. Las diferentes imágenes perceptuales que tenemos del mundo (perceptual) no contienen, entre ellas, nada que permita relacionar sus elementos unos con otros; esto es, en caso de que en ellas figure algún elemento que estaríamos dispuestos, de manera natural, a calificar como uno y el mismo objeto, Berkeley señalará que no hay tal relación óntica: los objetos son diferentes en tanto que figuran en imágenes perceptuales diferentes. 'La única relación es una verbal: decimos que se trata del mismo objeto porque esto facilita nuestra manera de tratar con los objetos del mundo perceptual.

Paso a considerar en detalle los puntos anteriores.

(a) Divisibilidad finita del objeto perceptual

Las anotaciones pertinentes en $\operatorname{los} P C$ para obtener el argumento de Berkeley, son:

En Geometria no se prueba que una pulgada sea divisible ad infinitum. (PC 247)

Supongamos que una pulgada representa una milla. 1/1000 de una pulgada es nada, pero $1 / 1000$ de la milla representada es algo por lo tanto 
1/1000 de una pulgada aun cuando es nada no es negligible, ya que representa algo esto es $1 / 1000$ de una milla. (PC 260)

Líneas particulares Determinadas no son divisibles ad infinitum, pero lo son las líneas como las usan los Geómetras ya que no se las determina a ningún número finito particular de puntos. Sin embargo un Geómetra (sin saber Él por qué) de muy buena gana dirá que puede demostrar que una línea de una pulgada es divisible ad infinitum. (PC 261)

Cuando una pequeña línea sobre Papel representa una milla los Matemáticos no calculan el l/1000 de la línea en el papel Calculan el $\mathrm{l} / 1000$ de la milla es esto lo que les importa, es en esto en lo que piensan si es que piensan o siquiera tienen alguna idea. La pulgada podría quizás representarles en su imaginación la milla pero el 1/1000 de pulgada no puede hacerse que represente algo ya que no es imaginable. (PC 341)

Comentando este pasaje, añade:

Pero siendo algo el 1/1000 de milla creen que es algo el 1/1000 de pulgada, cuando piensan en aquél imaginan que piensan en éste. ( $P C$ 34la)

En los Principios, Berkeley recoge las observaciones que ha expresado en los pasajes que acabamos de citar y las comenta de manera más amplia:

En otro lugar se ha observado que los teoremas y las demostraciones en la geometría se refieren a ideas universales. ...De aquí se sigue que de una línea de una pulgada en el dibujo debe hablarse como si contuviese diez mil partes, puesto que no se la considera en sí misma, sino en tanto que universal; y es universal sólo en su significación, por lo cual representa innumerables líneas mayores que ella misma, en las que pueden distinguirse diez mil partes o más, aun cuando en ella pueda no haber más de una pulgada. De esta manera, las propiedades de las líneas significadas (mediante una figura muy usual) se transfieren al signo y así, por error, se piensa que pertenecen a éste por su propia naturaleza. ( $P C H$, sec. 126)

$y$, continuando con el tema, en la sección siguiente leemos:

Porque no hay un número de partes tan grande, que no pueda haber una línea que contenga más, se dice que la línea de una pulgada contiene muchas más partes que cualquier número asignable de éstas, lo que es verdad no de la pulgada tomada en absoluto, sino de las cosas que significa. Pero, al no retener esta distinción en su pensamiento, los hombres se deslizan hacia la creencia de que la pequeña línea particular 
dibujada sobre el papel contiene, en sí misma, innumerables partes. (PCH, sec. 127)

Finalmente, en la siguiente sección leemos:

Por lo que se ha dicho, es clara la razón por la cual, para que cualquier teorema pueda hacerse universal en su uso, es necesario que hablemos de las líneas dibujadas sobre el papel como si contuviesen partes que realmente no contienen. Al hacer esto, si examinamos el asunto cuidadosamente, descubriremos, quizá, que no podemos concebir que una pulgada misma conste de, o sea divisible en, mil partes, sino sólo alguna otra línea mucho mayor de una pulgada y que ésta representa. (PCH, sec. 128)

Como conclusión, Berkeley, en los $P C$, afirmó:

Muy ciertamente ninguna Extensión finita divisible ad infinitum. (PC 314)

De los pasajes anteriores, podemos extraer el siguiente argumento berkeleyano en contra de la divisibilidad infinita: las extensiones perceptuales, finitas (para Berkeley, las únicas que hay), limitadas, están compuestas de un número finito de partes y no podemos, salvo sólo mediante un pensamiento equivocado, suponer que poseen más partes que las que realmente tienen. El argumento de Berkeley podemos verlo fundado en el siguiente principio:

$\left(A_{2}\right)$ Lo finito no es infinito.

Cieretamente, los matemáticos contemporáneos de Berkeley no ponían esto en cuestión, ${ }^{8}$ pero a Berkeley le extrañaba que, a pesar de que $\left(A_{2}\right)$ fuera un principio obvio y de que, conforme él veía las cosas, fuera aún más obvio que una línea de una pulgada es una línea de extensión finita y per-

8 "De manera explícita", habria que agregar, pues en $P C H, 123$, Berkeley señala to siguiente:

Después de los números pasamos a hablar de la extensión, la cual, considerada como relativa, es el objeto de la geometría. La divisibilidad infinita de la extensión finita, aun cuando no se la exprese abiertamente, sea como axioma o como teorema, en los elementos de esa ciencia, está, sin embargo, supuesta de igual manera en toda ella y se cree que tiene una conexión tan inseparable y esencial con los principios y demostraciones de la geometría, que los matemáticos nunca la ponen en duda ni la objetan en manera alguna.

Lo anterior pone una vez más de manifiesto que no es trivial enunciar trivialidades aun cuando, una vez enunciadas, pueda ser difícil ver dónde y cómo aplicarlas. Para mayores detalles acerca del asunto aquí tratado, cfr. supra n. 2. 
fectamente determinada, los matemáticos supusieran, sin embargo, que una línea así podía dividirse en un número infinito de partes.

Berkeley intenta dar una explicación a su extrañeza señalando una confusión en las consideraciones que los matemáticos hacían acerca de la línea de una pulgada dibujada en el papel. Consideraban, según él mismo señala, que sus argumentos se aplicaban a la línea en sí cuando, en realidad, deberian tomar esta línea como representante de cualquier otra línea, de la longitud que fuese, que muy bien pudiera dividirse en las partes que deseaban. ${ }^{\ominus}$

Ahora bien, en algunos de los pasajes citados, Berkeley argumenta en términos de la posibilidad de imaginar o no una cosa, de manera que parece vulnerable a una refutación empírica. La posibilidad que cada ser humano tiene de imaginar cosas puede variar mucho de uno a otro y así, si Berkeley no puede imaginar $1 / 1000$ de una pulgada esto no es argumento bastante para concluir que, en general, tal cosa no es imaginable. Alguien podría imaginarla. No obstante, en lo que ciertamente Berkeley tiene razón es en señalar que nadie puede imaginar - ni tener una representación mental cualquiera de - un infinito de partes dado como una totalidad. La idea misma de imaginar un conjunto inacabable como algo acabado, ciertamente encierra una contradicción. Berkeley nos dice:

¿Me preguntas si puede haber una idea infinita? Respondo que en un sentido puede haberla. así la esfera visual aun cuando sea tan pequeña

9 Russell tenía la idea, también, de que los enunciados y las demostraciones matemáticas y geométricas se referian a universales; eran éstos sus objetos de estudio propio y, según lo señaló de manera explícita, el estudio de las matemáticas imponía el estudio de los universales. En [17], p. 77, leemos:

Toda la matemática pura es a priori, como la lógica.

$[\ldots]$

Esto mismo lo ejemplifica la geometria. Si deseamos probar alguna propiedad de todos los triángulos, dibujamos algún triángulo y razonamos acerca de él; pero podemos evitar hacer uso de cualquier propiedad que no comparta con todos los otros triángulos y así, a partir de nuestro caso particular, obtenemos un resultado general.

$Y$ más adelante, en pp. 89-90, señala:

El hecho parece ser que todo nuestro conocimiento a priori se refiere a entidades que, hablando con propiedad, no existen ni en el mundo mental ni en el físico.

En un artículo publicado en 1911, "L'importance philosophique de la logistique", traducido luego como "The Philosophical Implications of Mathematical Logic" leemos:

La lógica y la matemática nos obligan, entonces, a admitir un tipo de realismo en el sentido escolástico, es decir, a admitir que hay un mundo de universales y de verdades que no inciden directamente sobre tal y cual existencia particular. ([15], p. 293.)

Lo que resulta importante recordar aquí es que, en lo tocante a los universales, las posiciones de Berkeley y Russell se oponian plenamente. 
es infinita. esto es no tiene terminación. Pero si por infinito quieres decir una extensión que conste de innumerables puntos. entonces te suplico me perdones. los puntos por muchos que sean pueden numerarse la multitud de puntos o pies, pulgadas, etc no impide en lo

* más mínimo su enumerabilidad. Muchos o la mayoría son enumerables así como unos cuantos o los menos. también si por idea infinita. quieres decir una idea demasiado grande para abarcarse o percibirse completa de una vez. debes excusarme. Pienso que tal infinito no es menos que una contradicción. ( $P C$ 475)

* esto es no impide que sean nombrables. (PC 475a)

En lo que aquí se ha dicho, no está en discusión la posibilidad de postular un infinito potencial o bien uno actual, sino sólo la posibilidad de que pueda presentarse una infinitud de puntos como una totalidad.10

De los pasajes anteriores podemos extraer el siguiente argumento berkeleyano:

Una totalidad infinita no es algo que pueda darse a la percepción o a la imaginación.

Una línea de una pulgada puede darse como totalidad a la percepción o a la imaginación.

por tanto:

Una línea de una pulgada no es una totalidad infinita. ${ }^{11}$

10 Cfr. supra, n. 4. Para una amplia y penetrante elaboración reciente de este argumento, cfr. "The Infinite in Mathematics", en [9], pp. 141-162.

11 Dos observaciones pertinentes a este respecto son las siguientes: Hobbes ya había defendido una doctrina similar y Zenón, según señala Moulines ([11], pp. 5-6), formuló una prueba indirecta acerca de la no divisibilidad infinita del espacio. La estructura de la prueba de Zenón, en la versión de Moulines, se aplica sin cambio (aun cuando matizaremos esta observación más adelante) al Berkeley de los $P C$. Veamos en detalle, ahora, a cada una de las dos consideraciones anteriores.

En [7], pp. 31-2, Hobbes nos dice:

Infinito. Cualquier cosa que imaginemos es finita. Por tanto, no hay idea o concepto de cosa alguna que llamemos infinito. Nadie puede tener en su mente una imagen de magnitud infinita ni de rapidez infinita, tiempo infinito, fuerza infinita o poder infinito. Cuando decimos de cualquier cosa que es infinita, sólo queremos decir que no somos capaces de concebir los extremos y límites de las cosas nombradas, no teniendo concepción alguna de la cosa, sino de nuestra propia inhabilidad... y también porque, como ya lo he dicho, cualquier cosa que concibamos primero se ha percibido, en todo o en parte, por los sentidos, un hombre no puede tener pensamiento alguno de nada que no se sujete a los sentidos. Nadie puede, pues, concebir nada que no conciba en algún lugar, dotado de alguna magnitud determinada y que pueda dividirse en partes; ni que cosa alguna se encuentre toda en un lugar y toda 
Lo que aqui podemos añadir es que esta conclusión de Berkeley entra en conflicto con el principio $\left(A_{1}\right)$, pues éste habla indiscriminadamente de una composición de partes extensas cuando lo que debería haber expresado es que tal composición debería ser, en principio, posible, ya que empírica-

en otro lugar al mismo tiempo, ni que dos o más cosas puedan estar en uno y el mismo lugar a la vez, pues nada de esto sucede jamás, ni puede incidir en los sentidos, sino que son discursos absurdos, tomados de oidas, sin significado alguno, de filósofos engañados y de escolásticos engañados o engañadores.

Lo que importa señalar aquí es la completa identificación de las tesis de Berkeley y las de Hobbes sobre el infinito, así como también el aparente rechazo que hace Hobbes de tesis escolásticas sobre la naturaleza de los universales. Berkeley estaría plenamente de acuerdo, también, con el rechazo de una tesis así; ciertamente conocia las obras de Hobbes, pero en los $P C$ (cfr. $P C$ 17, 795-9, 806, 822, 824-5, 827, 834, 837) cita o nombra a Hobbes (o al hobbismo) únicamente para criticarlo, sin hacer mención de estas doctrinas en las que hay coincidencia. Quizás Berkeley consideraba el materialismo de Hobbes demasiado peligroso como para encontrarle elementos positivos, ya que tal posición materialista, conforme a Berkeley, daría pie a los más crasos errores que él estaba luchando por erradicar. Lo que también interesa señalar es que Luce, p. ej., no nos ilustra demasiado acerca de este asunto concretándose a señalar que "Berkeley temía el materialismo de Hobbes, el que achaca a la separación entre esse y percipi; pero los dos pensadores tenfan puntos en común, por ejemplo, ambos rechazaban la 'idea de Dios' y compartían tesis similares acerca de los problemas matemáticos y acerca de los términos generales". (Cfr. [3], p. 454.) No entraré aquí a presentar una exégesis histórica de este asunto; sólo me contentaré con señalarlo.

Pasando al segundo tema al que aludí al principio de esta nota —el argumento de Zenón contra la divisibilidad infinita del espacio-, Moulines (loc. cit., p. 5), lo formula de la siguiente manera:

Teorema: El espacio no es infinitamente divisible.

Prueba: 1. Sea $S$ un intervalo cualquìera de magnitud finita.

2. Supongamos que el espacio es infinitamente divisible.

3. En tal caso, $S$ estará constituído por un número infinito de subintervalos espaciales $S_{1}$.

4. La magnitud de $S$ será igual a la magnitud de la suma de todos los $S_{1}$.

5. Luego la magnitud de $S$ será infinita, contra lo supuesto.

6. Esta contradicción muestra que el supuesto de que el espacio es infinitamente divisible es falso.

Aquí vale la pena señalar que un abogado de la divisibilidad infinita del espacio no tiene por qué conceder que del argumento se siga una contradicción sino que señalaría, en contra de Zenón, que o bien el argumento está incompletamente formulado o el punto mismo de partida, 1, es expresamente falso. Lo que explícitamente diria, por ejemplo, es que 1 tendria que formularse como:

1'. Sea $S$ un intervalo de magnitud finita (perceptible)

y la magnitud infinita a la que llega el argumento podria perfectamente ser no perceptible $y$ no habría contradicción aparente. Por otra parte, si la anterior no resulta una réplica: admisible (por alguna razón que habría que explicitar), podría señalarse que el supuesto 1 no puede admitirlo un defensor de la divisibilidad infinita, ya que para el no habría inter-valos finitos; el argumento, pues, sería una petitio en contra del defensor de la divisibilidad! infinita. Así, conforme a la primera réplica, habría que mostrar (lo que hace Berkeley dentro de su sistema) que no hay entidades no perceptibles y, en lo tocante a la segunda, 
mente podría suceder que algo extenso surja de algo no extenso. ${ }^{12}$ Berkeley, de manera clara, señala que una extensión perceptual tiene un límite finito de posibilidades de ser dividida y de seguir siendo perceptible; pasado ese límite, deja de tener esta propiedad y por esto, conforme a Berkeley, deja de ser. Ampliaré esta observación más adelante.

Si lo último que he apuntado es correcto, puede concluirse que el principio indiscriminado $\left(A_{1}\right)$ es falso. El énfasis de Berkeley es correcto, entonces. La divisibilidad indefinida puede, en principio, darse, pero empíricamente podrá no ser posible. Una línea física de una pulgada de longitud podrá dividirse hasta donde sea empíricamente posible hacerlo y no más allá. De cualquier forma, esta divisibilidad tendrá una terminación finita.

El alegato de Berkeley podemos verlo, entonces, como enfatizando un punto de gran importancia que no era tomado muy en cuenta en su época, a saber, que hay una gran diferencia entre lo que puede hacerse en principio (matemáticamente) y lo que puede hacerse empiricamente. ${ }^{13} \mathrm{La}$ divisibilidad infinita pertenece al mundo matemático (si es que pertenece a alguno), que no hay que confundir con el mundo de la experiencia. .

Russell, acerca de cuestiones como las anteriores, señaló lo siguiente:

No tenemos razones, sino prejuicios, para creer en la extensión infinita del espacio o del tiempo, al menos si consideramos espacio y tiempo como hechos físicos y no como ficciones matemáticas. Consideramos, naturalmente, espacio y tiempo como continuos, o al menos como compactos; pero, esto, a su vez, es más que nada un prejuicio. ${ }^{14}$

lo que quedaría por hacer (cosa que también encontramos en Berkeley) es mostrar que una posición asi (de infinita divisibilidad) engendra contradicciones, y sostener tal posición acerca del espacio físico sólo se debe a una confusión acerca del campo de aplicación de la divisibilidad ad infinitum que puede lograrse considerando "entidades" matemáticas.

12 Lo que de hecho parece suceder, conforme a las teorias de la fisica de particulas contemporánea, derivadas de la relatividad general einsteiniana, que señalan que no hay discontinuidad entre energia y partículas. $O$, dicho de manera más dramática o descriptiva, que consideran la energía una forma sutil de la materia.

13 Esta afirmación, sin embargo, no puedo hacerla con toda generalidad. A. C. Crombie, en [5], p. 28, ha señalado de manera clara que ya en el siglo xvu habia quienes tenian una idea precisa de esta distinción:

Descartes se propuso producir una ciencia verdadera de la naturaleza en la que todo se siguiese matemáticamente de primeros principios evidentes de suyo. La física moderna, claro está, rechaza la idea de que los principios de la física puedan ser evidentemente ciertos. Incluso en el siglo xvi Pascal y Huygens hicieron la misma crítica. Sefialaron que hay una diferencia esencial entre la física. y la matemática abstracta en tanto que los principios de la física, que exploran lo desconocido en el mundo concreto de hechos, están siempre expuestos a una refutación completa o parcial debida al descubrimiento de nuevos hechos.

14 [16], p. 140. 
Concluimos este apartado expresando nuestra convicción de que Berkeley, en los puntos que aquí hemos considerado, logra de manera satisfactoria minar las bases de una interpretación de los argumentos y enunciados matemáticos que los considerara enunciados descriptivos de la realidad perceptual, lo cual, a su vez, elimina el argumento más fuerte para fundar la composición en un número infinito de partes de los objetos extensos. ${ }^{15}$

Una vez señalado lo anterior, pasaremos a ver cuál es la que Berkeley considera la constitución de los objetos extensos de percepción.

\section{B) Minima sensibilia}

Acerca de la constitución de los objetos extensos perceptuales, en términos de minima sensibilia ( $m . s$. ), no encontramos en Berkeley observaciones tan claras y precisas como en el caso de la discusión anterior. Aqú intentaremos reconstruir las tesis de Berkeley en base a las observaciones que al respecto nos ha dejado en los $P C$.

Ciertamente, Berkeley poco nos dice acerca de los m.s. en sus obras publicadas; según señala Luce, ${ }^{16}$ "probablemente pretendía emplearlos en su proyectada obra matemática, como parte de su tratamiento de los infinitesimales, y

15 Moulines (en [11], p. 11), hablando de Epicuro, introduce la distinción entre una "métrica física y una teoría métrica abstracta (puramente matemeática)" $\mathrm{y}$, comentando la distinción, dice (ibid., nota al pie): "En realidad nos ha costado mucho llegar a esta distinción, e incluso después del advenimiento de las geometrías no-euclídeas, se han dado y se siguen dando confusiones a este respecto en la obra de eminentes filósofos y cientificos." Esta observación, con la que concuerdo plenamente, nos hace ver la distinción de Berkeley como un gran paso en la dirección correcta. Si bien puede señalarse que, en Berkeley, la distinción obedece a motivaciones teóricas que pueden ser difíciles de defender y, quizá por esta razón, no ha recibido la atención que merece, sin embargo, la distinción está ahf. Berkeley fue plenamente consciente de ella y la mantuvo en todos sus escritos $y$, de manera brillante, en su ataque expreso a los infinitesimales, en 1734, con la publicacion de The Analyst. Cfr., además, supra, n. 13.

16 [3], p. 333. En sus notas, Luce señala que el origen histórico de la noción berkeleyana de los minima se encuentra en la doctrina de los indivisibles de Cavalieri (ibid.). Más adelante nos presenta una breve semblanza biográfica de este autor, la que transcribo a continuación:

Bonaventura Cavalieri (1598-1647), profesor de matemáticas en Bolonia, escribió Geo-. metria Indivisibilibus Continuorum nova quadam ratione promota (1635) y Exercitationes Geometricae Sex (1647). En esta última obra expone sus métodos, anteriores y posteriores, y defiende su geometria de indivisibles contra los ataques de Guldinus. Imagina el continuo como compuesto de un número infinito de elementos últimos - moléculas que denomina indivisibles y generalmente se cree que sastuvo que los cuerpos están compuestos por una infinidad de superficies yuxtapuestas y las superficies compuestas de líneas. Berkeley estuvo de acuerdo con Cavalieri acerca de la existencia de indivisibles, pero difirió de él en cuanto al número de indivisibles en una línea finita. Berkeley sostuvo que el número es finito, lo que aparentemente negó Cavalieri. (Ibid. p. 386)

Vale la pena hacer resaltar aqui la importancia que Cavalieri tuvo en su momento como 
no deseaba sobrecargar su exposición del inmaterialismo con asuntos debatibles".

Las expresiones que Berkeley usa para referirse de manera especifica a los minima sensibilia en caso de que se trate de sensibles visuales o tangibles son, respectivamente, minimum visibile (m.v.) y minimum tangibile (m.t.).

La doctrina de Berkeley acerca de los m.s., en general, puede formularse en los siguientes términos: los m.s. son los elementos sensibles últimos; son átomos perceptuales, en sentido literal, que conforman cualquier objeto per. ceptual. Ahora bien, en cualquier presentación perceptual está presente sólo un número finito de tales átomos. Todos ellos son perceptibles, aun cuando no se den como individuos aislados, sino conformando la presentación total. Es importante recordar una vez más que una de las motivaciones más fuertes de Berkeley para ir en contra de la tesis de la divisibilidad infinita y postular la composición finita del objeto de percepción es que la tesis mencionada va en contra de su principio "esse est percipi", ya que sería imposible, según hemos argumentado anteriormente, que todos los puntos infinitos que, siguiendo tal tesis, conforman cualquier objeto extenso de percepción, sean perceptibles. Por otra parte, podemos añadir que los m.s. surgen como resultado de la anterior discusión de Berkeley en contra de la divisibilidad infinita de los objetos de percepción: si un objeto de percepción es sólo finitamente divisible, esta divisibilidad llega, entonces, a un punto pasado el cual ya no es posible dividir más el objeto perceptual dado y obtener como residuo un objeto perceptual nuevo. Son justamente estos límites últimos de perceptibilidad los que Berkeley denomina m.s. Para obtener los m.s. no importa cuál sea el método que empleemos al efectuar la división del objeto perceptual dado; de cualquier manera obtendremos m.s. finitos en número. Además, siempre habrá el mismo número de m.s. en la presentación total.

Para tener un marco de referencia acerca de la tesis general contra la que Berkeley formula su propia tesis de los m.s., me parece conveniente abrir aquí un paréntesis y considerar las ideas que Locke formula en su Ensayo sobre la constitución última de los objetos materiales del mundo exterior.

precursor del cálculo, citando los comentarios que, acerca de él, han vertido dos destacados historiadores de la matemática:

Veinte años después de la publicación de la Stereometria doliorum de Kepler, apareció en Italia un libro que fue su rival en popularidad. Tan famosa se hizo la Geometria Indivisibilibus de Bonaventura Cavalieri, que se ha mantenido, con alguna justicia, que el nuevo análisis surgió de la aparición de este libro en 1635. ([2], p. 111.)

Y también:

Un método de diferenciación y de integración, derivado de la comprensión plena de que un proceso es el inverso del otro, sólo podían descubritlo hombres que dominasen el método geométrico de los griegos y de Cavallieri, así como el método algebraico de Descartes y Wallis. ([18], p. 109.) 


\section{a) Locke y las particulas insensibles 17}

En el libro II del Ensayo, Locke aborda de manera puramente mecanicista la relación que se da entre los cuerpos externos y el sujeto que los percibe mediante ideas. Explica la producción de las ideas en el sujeto mediante el choque de partículas materiales dotadas de sus cualidades primarias, siendo las de movimiento-y-reposo y solidez las que de manera sobresaliente actúan en este caso. Al chocar con las terminaciones nerviosas del sujeto, esas partículas de materia producirán, de alguna manera que Locke no encuentra forma de explicar, las ideas de las cualidades de los cuerpos.

Ahora bien, quizás esta explicación (parcial) pueda dar cuenta de la forma como percibimos los objetos mediante el tacto, pero no es obvio, en manera alguna, cómo aclara, por ejemplo, los perceptos visuales producidos por los objetos; esto es, como da razón de los perceptos de objetos que se encuentran a alguna distancia de nosotros.

Para dar respuesta al problema anterior, Locke apela a "particulas insensibles" de materia que salen del objeto como "emisarios" del mismo y, al chocar con nuestros órganos de la visión, producen los perceptos visibles.

La manera como formula esto es la siguiente:

Lo que hemos de considerar de inmediato es cómo los cuerpos producen ideas en nosotros y, manifiestamente, la única forma como podemos concebir que operan los cuerpos es por impulso.

Si, entonces, los objetos externos no están unidos a nuestras mentes cuando producen ideas en ellas y, sin embargo, percibimos estas cualidades originales en aquéllos que singularmente caen bajo nuestros sentidos, es evidente que por esta razón nuestros nervios, nuestros espíritus animales o algunas partes de nuestros cuerpos conduzcan algún movimiento al cerebro o al asiento de la sensación para producir en nuestras mentes las ideas particulares que tenemos de ellos. $\mathrm{Y}$ puesto que la extensión, la figura, el número y el movimiento de los cuerpos de una grandeza observable puede percibirlos la vista a la distancia, es evidente que algunos cuerpos, singularmente imperceptibles, deben comunicar algún movimiento al cerebro, movimiento que produce en nosotros estas ideas que tenemos de ellos.

De la misma manera como se producen en nosotros las ideas de estas cualidades originales, podemos concebir que también se producen las ideas de las cualidades secundarias, esto es, por la operación, sobre nuestros sentidos, de partículas insensibles. Pues es manifiesto que hay cuerpos, y una buena cantidad de ellos, que son, cada uno de ellos, tan pequeños que de los mismos no podemos descubrir, mediante ninguno de nuestros sentidos, su volumen, figura o movimiento. ([10], II, viii, 11, 12, 13.)

1) Gran parte de lo que se presenta en este inciso lo tomé de [14], pp. 3-12. 
Puesto que la única forma que Locke tiene a la mano para explicar la génesis de las ideas apela a las cualidades primarias de los cuerpos (de las partículas insensibles de los cuerpos, para ser más precisos) - lo cual parece permitirle evitar los problemas de la acción a distancia, que la física mecanicista había intentado resolver-, tendrá que mantener que cualquier idea sensible, aun las ideas no táctiles, es producida por el choque de particulas sólidas de materia con las terminaciones nerviosas de los órganos de sensación. Ahora bien, es inútil buscar en Locke una explicación de la transformación de estas alteraciones motoras en ideas; él mismo señala su extrañeza y confiesa su ignorancia diciendo:

No es mayormente imposible concebir que Dios anexase tales ideas a tales movimientos con los que no tienen similitud alguna, que concebir que haya unido la idea de dolor al movimiento de un pedazo de acero al dividir nuestra piel, con el que aquella idea no tiene semejanza alguna. ([10], II, viii, 13.)

En su libro Examination of Malebranche, nos dice, de manera más directa:

Creo que entiendo las impresiones que los rayos de luz hacen sobre la retina; así como también pueden concebirse los movimientos que de allí prosiguen hasta el cerebro. $\mathrm{Y}$ estoy persuadido de que éstos producen ideas en nuestras mentes, pero de una forma que para mí es incomprensible. Esto lo dejo a la buena disposición de Dios, cuyos caminos rebasan nuestra comprension. ${ }^{18}$

Aun sin detenernos más en este problema, de lo que he dicho anteriormente parece obtenerse una conclusión bastante extraña: que Ia solidez —según Locke, una cualidad que sólo se percibe por el tacto- sería, conforme a la tesis de las partículas insensibles, una propiedad común a todos los sentidos.

Locke, para explicar qué es lo que me permite decir con verdad, por ejemplo, "Veo ahora un tintero", tendrá que señalar que la idea del tintero que ahora se me presenta, se origina debido a la emisión de partículas "insensibles" desde el tintero hasta mis ojos. Las partículas en cuestión son insensibles por la sencilla razón de que no me es posible verlas ni sentirlas, pero esto no excluye que las mismas tengan cualidades primarias lockeanas (o algo parecido a éstas, según explicaremos de inmediato), ni que puedan producir efectos en nosotros.

$\mathrm{La}$ introducción de las partículas insensibles trae aparejados otros problemas en la explicación epistemológica. Dado que son insensibles, cada una 
de ellas por sí sola, es imposible que conozcamos sus cualidades particulares; de tales cualidades sólo podemos hablar en términos generales en el sentido de decir que las particulas insensibles deben poseer cualidades primarias, pero sin que podamos decir cuáles y cómo son tales cualidades. Locke nos habla de

... esta ignorancia que tenemos acerca de las cualidades primarias de las partes insensibles de los cuerpos ([10], IV, iii, 12)

y no sólo esto, sino que además añade:

$Y$ por tanto estoy dispuesto a dudar de que por mucho que el ingenio humano pueda avanzar en la filosofia utilitaria y experimental acerca de las cosas físicas, la cientifica estará aún fuera de nuestro alcance... ([10], IV, iii, 26).

Pero si, conforme a la tesis de Locke, la constitución última, real, de un cuerpo está fundada en partículas insensibles de las que no tenemos conocimiento cierto, entonces podemos concluir que nuestras ideas de percepción del mundo que nos rodea no nos presentan a los cuerpos tal como son. Pareceria posible, así, concluir aquí con una nota pesimista acerca de nuestro conocimiento del mundo físico: conforme a las tesis de Locke (que aquí apenas hemos esbozado), nuestro (supuesto) conocimiento del mundo físico es una ilusión constante e irremediable. De alguna manera podemos decir que nuestras ideas de las cualidades primarias de los cuerpos sólo nos presentan las cualidades que, siguiendo a Jackson,19 denominaré "macroscópicas", pero que las cualidades reales de tales cuerpos, las de sus minúsculas partes insensibles, quedarán siempre fuera de nuestro alcance cognoscitivo.

De ser éstas las conclusiones que pueden sacarse de las tesis del que Berkeley consideraba el mayor filósofo de su tiempo - y éste mismo las presenta de manera explícita, según hemos visto-, a lo único que se puede llegar es al escepticismo. Berkeley no era escéptico en manera alguna y deseaba luchar denodadamente contra tal posición. Por esto, en muchas de las anotaciones de sus $P C$, le escuchamos afirmar jubilosamente que de sus principios no se llega al escepticismo, sino que se adquiere un conocimiento directo y cierto de las cosas. Así, nos dice:

Tengo una certeza mayor que Mr. Locke acerca de la realidad y existencia de los Cuerpos puesto que él sólo pretende tener lo que llama conocimiento sensorial, en tanto que yo creo tener conocimiento demostrativo de su Existencia, entendiendo por ello combinaciones de poderes en un sustrato desconocido. (PC 80)

19 Cfr. [8], p. 66. 
Más adelante:

El Reverso del Principio introdujo el Escepticismo. ( $P C$ 304)

E insiste nuevamente:

Yo estoy más alejado del escepticismo que cualquier. hombre. Conozco con un conocimiento intuitivo la existencia de otras cosas así como mi propia Alma. esto es lo que ni Locke ni escasamente cualquier otro Filósofo Pensante aspira a tener. ( $P C$ 563)

Contra este telón de fondo polémico han de aclararse muchas de las afirmaciones $y$ tesis de Berkeley que vamos a considerar en la segunda parte de este escrito.

J. A. Robles

Instituto de Investigaciones Filosóficas

Universidad Nacional Autónoma de México

\section{BIBLIOGRAFIA}

[1] Borges, J. L.: "La doctrina de los ciclos", en Historia de la eternidad. Emecé Editores, S. A., Buenos Aires, 1953; pp. 75-89.

[2] Boyer, C. B.: The History of the Calculus and its Conceptual Development (1949). Dover Publications Inc., Nueva York, 1959.

[3] Berkeley, G.: Philosophical Commentaries generally called The Commonplace Book; una editio diplomatica transcrita y editada con una introducción y notas por A. A. Luce MC DD Litt D. Thomas Nelson and Sons Limited; Londres, 1944. (Agradezco las facilidades que me fueron otorgadas por el maestro Raúl Quesada, durante su gestión como Secretario Académico del Instituto de Investi. gaciones Filosóficas, para la obtención de esta edición, y a la maestra Gertrude Distel, a cargo entonces de las adquisiciones bibliotecarias del mencionado Instituto, quien facilitó los trámites para que pudiera tener rápidamente esta obra a mi disposición.)

[4] —: The Works of George Berkeley Bishop of Cloyne; Vols. I-IX. Edición de A. A. Luce y T. E. Jessop. Thomas Nelson and Sons, Ltd., Londres, 19481957.

[5] Crombie, A. C.: "Descartes" [1959]; en Mathematics, an Introduction to its Spirit and Use (with an Introduction by M. Kline). Readings from Scientific American; W. H. Freeman and Co., San Francisco, Cal., 1979; pp. 22-8.

[6] Greek Mathematical Works I (Thales to Euclid), Ivor Thomas (trad.); Loeb Classical Library. Harvard University Press, Cambridge, Mass., 1967.

[7] Hobbes, Th.: Leviathan: or the Matter, Forme and Power of a Commonwealth Ecclesiasticall and Civil. Selected, and with an Introduction, by Richard S. Pet- 
ers; edited by Michael Oakeshott. Collier Books, Collier-Macmillan Ltd., Londres, 1969.

[8] Jackson, R.: "Locke's Distinction between Primary and Secondary Qualities"; en Locke and Berkeley: A Collection of Critical Essays; edición de C. B. Martin y D. M. Armstrong. Anchor Books; Doubleday \& company, Inc., Nueva York, 1968.

[9] Lazerowitz, M.: The Language of Philosophy. D. Reidel Publishing Company, Dordrecht, Holanda, 1977.

[10] Locke, J.: An Essay Concerning Human Understanding; cotejado y anotado, con prolegómenos biográfico, crítico e histórico, por Alexander Campbell Fraser. Dover, Nueva York, 1959.

[11] Moulines, C. U.: "Sobre el problema del 'atomismo geométrico' en Epicuro", en Dianoia, Anuario de Filosofía, UNAM/FCE, México, 1977; pp. 1-12.

[12] O'Connor, D. J.: John Locke. Penguin Books, Londres, 1952.

[13] Robles, J. A.: "Borges, Cantor y el eterno retorno"; en Thesis, nueva revista de Filosofía y Letras, Núm. 4, enero 1980. UNAM, México; pp. 49-54.

[14] - Percepción y Significado en G. Berkeley; tesis de licenciatura, UNAM, 1967 (inédito).

[15] Russell, B.: Essays in Analysis; editado por Douglas Lackey. George Allen \& Unwin Ltd., Londres, 1973.

[16] —-: Introduction to Mathematical Philosophy (1919). Touchstone Books, Simon and Schuster, Nueva York, 1967.

[17] — : The Problem of Philosophy (1912). Oxford University Press, Londres, 1959.

[18] Struik, D. J.: A Concise History of Mathematics; third revised edition. Dover Publications Inc., Nueva York, 1967.

[19] Swift, J.: Gulliver's Travels. Dell Publishing Co., Inc., Nueva York, 1977. 